International Journal of Pure and Applied Mathematics

Volume 92 No. 4 2014, 525-534

ISSN: 1311-8080 (printed version); ISSN: 1314-3395 (on-line version)

url: http://www.ijpam.eu

doi: http://dx.doi.org/10.12732/ijpam.v92i4.7

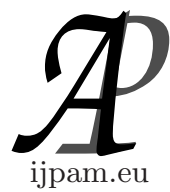

\title{
$(\alpha, 1)$ DERIVATIONS ON SEMIRINGS
}

\author{
S.P. Nirmala Devi ${ }^{1}$, M. Chandramouleeswaran ${ }^{2} \S$ \\ ${ }^{1}$ Sree Sowdambika College of Engineering Aruppukottai, INDIA \\ ${ }^{2}$ Department of Mathematics \\ Saiva Bhanu Kshatriya College \\ Aruppukottai, 626101, INDIA
}

\begin{abstract}
Motivated by some works on derivations on rings and near-rings, Chandramoulesswaran and Thiruveni discussed the notion of derivations on semirings. In this paper, we introduce the notion of two sided $\alpha$ derivation and $(\alpha, 1)$ derivation on a semiring and derive some of its properties.
\end{abstract}

AMS Subject Classification: 16 Y 30

Key Words: prime semiring, semiprime semiring, $(\alpha, 1)$ derivation

\section{Introduction}

The notion of a semiring was introduced by H.S. Vandiver [6] in 1934. A semiring is a universal algebra with two associative binary operations, where one of them distributes over the other. Though more general definitions are sometimes used, for this paper a semiring will be defined to be a set $S$ with two operations + (addition) and (multiplication); with respect to addition, $S$ is a commutative monoid with 0 as its identity dement. With respect to multiplication, $S$ is a (generaily noncommutative) monoid with 1 as its identity element. Connecting the two algebraic structures are the distributive laws, $a .(b+c)=a . b+a . c$ and $(a+b) . c=a . c+b . c$ for all $a, b, c \in S$, and the requirement $a .0=0 . a=0$ for every $a \in S$.

Received: November 24, 2013

(C) 2014 Academic Publications, Ltd. url: www.acadpubl.eu

${ }^{\S}$ Correspondence author 
The notion of derivations of rings can be naturally extended in semirings (see [3], page 30). The theory of derivations of semirings is not well developed as compared to the theory of derivations of rings (see [5]) due to the absence of additive inverse and the lack of some important concepts included by commutators. This motivated Chandramouleeswaran and Thiruveni to discuss in detail the notion of derivations on semirings $[1,2]$. In their paper [4], the authors introduced the notion of two-sided $\Gamma-\alpha$ - derivations in prime and semiprime $\Gamma$-near-rings. In this paper, we introduce the notion of two sided $\alpha$ derivation and $(\alpha, 1)$ derivation on a semiring and derive some of its properties on prime semirings.

\section{Preliminaries}

In this section we recall some basic definitions on semirings and derivations on it, that are needed for our work.

Definition 2.1. A semiring $(S,+, \cdot)$ is an algebraic system with a nonempty set $S$ together with two binary operations + and $\cdot$ such that

1. $(S,+)$ is a semi group

2. $(S, \cdot)$ is a semi group

3. For all $a, b, c \in S, a \cdot(b+c)=a \cdot b+a \cdot c$ and $(a+b) \cdot c=a \cdot c+b \cdot c$ hold.

Definition 2.2. A semiring $(S,+, \cdot)$ is said to be additively commutative if $(S,+)$ is a commutative semi group. A semiring $(S,+, \cdot)$ is said to be multiplicatively commutative if $(S, \cdot)$ is a commutative semi group. It is said to be commutative if both $(S,+)$ and $(S, \cdot)$ are commutative.

Definition 2.3. The semiring $(S,+, \cdot)$ is said to be a semiring with zero, if it has an element 0 in $S$ such that $x+0=x=0+x$ and $x \cdot 0=0=0 \cdot x \quad \forall x \in S$.

Definition 2.4. A semiring $(S,+, \cdot)$ is said to be a semiring with an identity element 1 , if there exists an element $1 \neq 0 \in S$ such that $1 \cdot x=x=$ $x \cdot 1 \forall x \in S$.

Definition 2.5. Let $(S,+, \cdot)$ be a semiring. An element $\alpha$ of $\mathrm{S}$ is called additively left cancellative if for all $\alpha, \beta, \gamma \in S, \alpha+\beta=\alpha+\gamma \Rightarrow \beta=\gamma$. If every element of a semiring $S$ is additively left cancellative, it is called an additively left cancellative semiring.

Analogously, we define an additively right cancellative semiring. 
Definition 2.6. A semiring $(S,+, \cdot)$ is additively cancellative if it both additively left and right cancellative.

Definition 2.7. Let $(S,+, \cdot)$ be a semiring. A derivation on $S$ is a map $d: S \rightarrow S$ satisfying the following conditions

1. $d(x+y)=d(x)+d(y) \quad \forall x, y \in S$

2. $d(x y)=d(x) y+x d(y), \quad \forall x, y \in S$.

Example 2.8. Let $S$ be a semiring. Let $M_{2}(S)=\left\{\left(\begin{array}{cc}a & 0 \\ b & c\end{array}\right) \mid a, b, c \in S\right.$. $\}$ Define $d: M_{2}(S) \rightarrow M_{2}(S)$ is given by $d\left[\left(\begin{array}{ll}a & 0 \\ b & c\end{array}\right)\right]=\left(\begin{array}{ll}0 & 0 \\ b & 0\end{array}\right)$

Then $d$ is a derivation on $M_{2}(S)$.

Definition 2.9. Let $S$ be a semiring with centre $Z(S)$

1. $S$ is said to be prime if $a S b=0 \Rightarrow a=0$ or $b=0$.

2. $S$ is said to be semiprime if $a S a=0 \Rightarrow a=0$.

3. $S$ is said to be 2 torsion free if $2 a=0, a \in S \Rightarrow a=0$.

Definition 2.10. Let $S$ be a semiring. A left $S$-semimodule is a commutative monoid $(M,+)$ with additive identity $0_{M}$ for which we have a function $S \times M \rightarrow M$, denoted by $(s, m) \rightarrow s m$ and called scalar multiplication, which satisfies the following conditions for all elements $s$ and $s^{\prime}$ of $S$ and all elements $m$ and $m^{\prime}$ of $M$ :

1. $\left(s s^{\prime}\right) m=s\left(s^{\prime} m\right)$

2. $s\left(m+m^{\prime}\right)=s m+s m^{\prime}$

3. $\left(s+s^{\prime}\right) m=s m+s^{\prime} m$

4. $1_{S} m=m$

5. $s 0_{M}=0_{M}=0_{S} m$.

If $V(M)=M$ then $M$ is an $S$-module where $V(M)$ is the set of all elements of $M$ having additive inverse.

Definition 2.11. Let $(S,+, \cdot)$ be a semiring. Let $\alpha$ be endomorphism os $S$. An additive mapping $d: S \rightarrow X$ is said to be an $\alpha$ derivation if $d(x y)=$ $\alpha(x) d(y)+d(x) y$. 
Definition 2.12. Let $\alpha, \beta$ be two endomorphism on $S$. An additive mapping $d: S \rightarrow X$ is said to be an $(\alpha, \beta)$ derivation if $d(x y)=\alpha(x) d(y)+$ $d(x) \beta(y) \quad \forall x, y \in S$.

\section{3. $(\alpha, 1)$ Derivations}

In this section, we introduce the notion of $(\alpha, 1)$ derivations on semirings, to illustrate the concept, discuss examples and prove some simple properties.

Definition 3.1. Let $\alpha$ be an endomorphism on $S$. An additive map $d:$ Srightarrow $X$ is called a

1. $(\alpha, 1)$ derivation if $d(x y)=\alpha(x) d(y)+d(x) y$

2. $(1, \alpha)$ derivation if $d(x y)=x d(y)+d(x) \alpha(y)$

Definition 3.2. An additive map $d: S \rightarrow X$ is called a two sided $\alpha$ derivation if $\mathrm{d}$ is an $(\alpha, 1)$ derivation as well as $(1, \alpha)$ derivation.

Example 3.3. Let $S$ be commutative semiring.

Let $M_{2}(S)=\left\{\left(\begin{array}{ll}a & b \\ 0 & c\end{array}\right) \mid a, b, c \in S\right\}$

Define $d: M_{2}(S) \rightarrow M_{2}(S)$ and $\alpha: M_{2}(S) \rightarrow M_{2}(S)$ by

$$
d\left[\left(\begin{array}{ll}
a & b \\
0 & c
\end{array}\right)\right]=\left(\begin{array}{ll}
0 & b \\
0 & 0
\end{array}\right)
$$

and

$$
\alpha\left[\left(\begin{array}{ll}
a & b \\
0 & c
\end{array}\right)\right]=\left(\begin{array}{ll}
a & 0 \\
0 & c
\end{array}\right)
$$

respectively.

Then $d$ is a two sided $\alpha$ derivation.

Example 3.4. Let $\alpha_{1}: M_{2}(S) \rightarrow M_{2}(S)$ be given by

$$
\alpha_{1}\left(\left(\begin{array}{ll}
a & b \\
0 & c
\end{array}\right)\right)=\left(\begin{array}{ll}
a & 0 \\
0 & 0
\end{array}\right) .
$$

Then $d$ is an $\left(\alpha_{1}, 1\right)$ derivation but not a $\left(1, \alpha_{1}\right)$ derivation.

Example 3.5. Let $\alpha_{2}: M_{2}(S) \rightarrow M_{2}(S)$ be given by

$$
\alpha_{2}\left(\left(\begin{array}{ll}
a & b \\
0 & c
\end{array}\right)\right)=\left(\begin{array}{ll}
0 & 0 \\
0 & c
\end{array}\right) .
$$

Then $d$ is a $\left(1, \alpha_{2}\right)$ derivation but not an $\left(\alpha_{2}, 1\right)$ derivation. 
Example 3.6. Let $S_{1}$ and $S_{2}$ be two semirings and let $S: S_{1} \oplus S_{2}$. Let $d_{1}$ be any map on $S_{1}$ and $d_{2}$ be the left and right sub-module homomorphism on $S_{2}$. Define $d: S \rightarrow X$ by $d\left(s_{1}, s_{2}\right)=\left(0, d\left(s_{2}\right)\right)$ and $\alpha: S \rightarrow X$ by $\alpha\left(s_{1}, s_{2}\right)=$ $\left(d\left(s_{1}\right), 0\right)$. Then $d$ is a two sided $\alpha$ derivation but not a derivation.

Lemma 3.7. Let $S$ be prime semiring and $I$ be a non zero ideal of $S$. If $a+b-a-b=0 \forall x, y \in I$. Then $(S,+)$ is abelian.

Proof. We have

$$
a+b-a-b=0 \forall x, y \in I .
$$

Let $I$ be a non zero ideal of $S$. Then for all $x, y \in S$ and all $a \in I, x a, y a \in I$. Hence $(*)$ takes form:

1. $x a+y a-x a-y a=0$.

2. $(x+y-x-y) a=0 \forall x, y \in$ Sand $a \in I$.

3. $(x+y-x-y) I=0$.

4. $(x+y-x-y) S I=0$.

Since $I$ is an ideal, $I \neq 0$ and $S$ is a prime semiring $x+y-x-y=0 \forall x, y \in S$. Therefore $(S,+)$ is abelian.

Lemma 3.8. Let $S b$ a Semiring and $I$ be a Non zero ideal of $S$. Let $d$ be a Non zero $(\alpha, 1)$ derivation on $S$. If $d(x+y-x-y)=0 \quad \forall x, y \in I$, then $\alpha(x+y-x-y) d(z)=0 \quad \forall x, y, z \in I$.

Proof. Assume that $d(x+y-x-y)=0 \forall x, y \in I$.

Taking $y z$ and $x z$ instead of $y$ and $x$, we have

1. $d(x z+y z-x z-y z)=0$.

2. $d((x+y-x-y) z)=0$.

3. $\alpha(x+y-x-y) d(z)+d(x+y-x-y) z=0$

4. $\alpha(x+y-x-y) d(z)=0 \forall x, y \in I$.

Lemma 3.9. Let $S$ be a prime semiring and $I$ be a non zer ideal of $S$. let $\alpha$ be anon zero $(\alpha, 1)$ derivation on $S$. If $x \in S$ and $d(I) x=0$ then $x=0$.

Proof. Since $d(I) x=0$, we have

$$
\begin{aligned}
d(u a) x & =0 \forall a \in I, u \in S \\
(\alpha(u) d(a)+d(u) a) x & =0
\end{aligned}
$$




$$
\begin{aligned}
\alpha(u) d(a) x+d(u) a x & =0 \forall a \in I, \mid x \in S \\
d(u) a x & =0
\end{aligned}
$$

Replacing $u$ by $u v$, we have

$$
\begin{aligned}
d(u v) a x & =0 \\
(\alpha(u) d(v)+d(u) v) a x & =0 \\
\alpha(u) d(v) a x+d(u) v a x & =0 \\
d(u) \text { Sax } & =0 \\
d(u) S I x & =0
\end{aligned}
$$

Since $S$ is prime, $d(u)=0$ or $I x=0$. Since $d \neq 0, \quad I x=0$. Since $I \neq 0 \quad x=0$.

Theorem 3.10. Let $S$ be an additively cancellative semiring and $I$ a multiplicatively subsemigroup of $S$. Let $d$ be an $(\alpha, 1)$ derivation of $S$ and $\alpha(I)=I$.

1. If $d$ acts as a homomorphism on $I$ then

$$
d(x) y d(x)=d(x) y x=\alpha(x) y d(x) \quad \forall x, y \in I
$$

2. If $d$ acts as an antihomomorphism, then

$$
d(x) y d(x)=d(x) x y=y \alpha(x) d(x) \quad \forall x, y \quad \in I
$$

Proof. (1) Since $d$ is a $(\alpha, 1)$ derivation and it is a homomorphism we have

$$
d(x y)=\alpha(x) d(y)+d(x) y \quad \forall x, y \in S .
$$

Substitute yx for y in(1) we have

$$
\begin{aligned}
d(x y x) & =\alpha(x) d(y x)+d(x) y x \\
& =\alpha(x) d(y) d(x)+d(x) y x
\end{aligned}
$$

But

$$
d(x y x)=d(x y) d(x)=\alpha(x) d(y) d(x)+d(x) y d(x) .
$$

From (2) and (3), we have $d(x) y d(x)=d(x) y x$.

Replacing in (1): $x$ by $y x$, we obtain

$$
d(y x y)=\alpha(y x) d(y)+d(y x) y
$$




$$
=\alpha(y) \alpha(x) d(y)+d(y x) y .
$$

But $d(y x y)=d(y) d(x y)=d(y)(\alpha(x) d(y)+d(x) y)$, therefore

$$
\begin{aligned}
d(y x y) & =d(y) \alpha(x) d(y)+d(y) d(x) y \\
& =d(y) \alpha(x) d(y)+d(y x) y .
\end{aligned}
$$

From (4) and (5), we get $\alpha(y) \alpha(x) d(y)=d(y) \alpha(x) d(y)$.

Since $\alpha(I)=I, \alpha(y) w d(y)=d(y) w d(y) \forall y, w \in I$ we obtain $\alpha(x) y d(x)=$ $d(x) y d(x)$.

ii) Since $d$ is a $(\alpha, 1)$ derivation and it is an antihomomorphism, we have

$$
d(x y)=\alpha(x) d(y)+d(x) y=d(y) d(x) .
$$

Substitute $x y$ for $y$ we have

$$
\begin{aligned}
d(x x y) & =\alpha(x) d(x y)+d(x) x y . \\
& =\alpha(x) d(y) d(x)+d(x) x y \\
(\text { but } d(x x y) & =d(x y) d(x)) \\
& =(\alpha(x) d(y)+d(x) y) d(x)=\alpha(x) d(y) d(x)+d(x) y d(x) .
\end{aligned}
$$

From (6) and (7), we get $d(x) y d(x)=d(x) x y$.

Replace $x$ by $x y$ in $(1), \alpha(x y) d(y)+d(x y) y=d(y) d(x y)$.

$$
\begin{aligned}
d(x y y) & =\alpha(x y) d(y)+d(x y) y \\
& =\alpha(x) \alpha(y) d(y)+d(x y) y \\
(\text { but } d(x y y) & =d(y) d(x y)) \\
& =d(y)(\alpha(x) d(y)+d(x) y) \\
& =d(y) \alpha(x) d(y)+d(y) d(x) y \\
& =d(y) \alpha(x) d(y)+d(x y) y .
\end{aligned}
$$

(8) and (9) yields $\alpha(x) \alpha(y) d(y)=d(y) \alpha(x) d(y)$.

From $\alpha(I)=I, \alpha(x)=x$, we obtain $x \alpha(y) d(y)=d(y) x d(y) \quad \forall x, y \in I$.

Theorem 3.11. Let $S$ be an additively cancellative semiring and let $I$ be a ideal of $S$ which contain zero. Let $d$ be a twosided $\alpha$ derivation on $S$ such that $\alpha(I)=I$ and if $d$ acts as a homomirphism on $I$ then $d(I)=0$.

Proof. By the Theorem 1,

$$
d(x) y d(x)=\alpha(x) y d(x) \forall x, y \in I .
$$


Multiplying by $d(z)$ we have

$$
\begin{aligned}
d(z) d(x) y d(x) & =d(z) \alpha(x) y d(x) \\
d(z x) y d(x) & =d(z) \alpha(x) y d(x)
\end{aligned}
$$

Since $d$ is $(\alpha, 1)$ derivation, then

$$
\begin{aligned}
(z d(x)+d(z) \alpha(x)) y d(x) & =d(z) \alpha(x) y d(x), \\
z d(x) y d(x)+d(z) \alpha(x) y d(x) & =d(z) \alpha(x) y d(x), \\
z d(x) y d(x) & =0 .
\end{aligned}
$$

Taking $n y$ instread of $y$ in the equalities above, we derive

$$
\begin{array}{rlrl}
z d(x) n y d(x) & = & 0 \quad \forall x, y z \in I n \in S \\
z d(x) S y d(x) & = & & \\
\text { by primeness, } \begin{aligned}
y d(x) & =
\end{aligned} & 0 \quad \text { and } \quad \alpha(I)=I \\
\alpha(y) d(x) & = & & 0 \quad \forall x, y \in I .
\end{array}
$$

Substituing $n x$ for $x$ and multiplying in the right hand side by $d(y)$, we obtain

$$
\begin{aligned}
\alpha(y) d(n x) d(y) & =0 \\
\alpha(y)(n d(x)+d(n) \alpha(x)) d(y) & =0 \\
(\alpha(y) n d(x)+\alpha(y) d(n) \alpha(x)) d(y) & =0 \\
\alpha(y) n d(x) d(y)+\alpha(y) d(n) \alpha(x) d(y) & =0 \\
\alpha(y) n d(x) d(y)+y d(n) x d(y) & =0 \\
\forall x, y & \in I \\
\alpha(y) n d(x y) & =0 \\
\alpha(y) n(\alpha(x) d(y)+d(x) y) & =0 \\
\alpha(y) n \alpha(x) d(y)+\alpha(y) n d(x) y & =0 \\
\alpha(y) n d(x) y & =0 \\
\alpha(y) S d(x) y & =0 .
\end{aligned}
$$

By primeness

$$
(x) y=0 .
$$

Comparing (3) and (6), we derive

$$
\alpha(y) d(x)+\alpha(x) y=0
$$




$$
d(y x)=0
$$

Now, replace $y$ by $y n$ :

$$
\begin{aligned}
d(y n x) & =0 \quad \forall y \in I \quad \text { and } n \in S \\
d(y n x) & =0 \\
d(y) d(n x) & =0 \\
d(y)(n d(x)+d(n) \alpha(x)) & =0 \\
d(y) n d(x)+d(y) d(n) \alpha(x) & =0 \\
d(y) n d(x)+d(y n) d(x) & =0 \\
d(y) n d(x) & =0 \\
d(x) & =0 \quad \forall x \in I
\end{aligned}
$$

Corollary 3.12. Let $S$ be a prime semiring and $I$ be a semigroup ideal of $S$ containing zero. Let $d$ be a twosided $\alpha$ derivation on $S$ such that $\alpha(I)=I$ and if $d$ acts as a homomorphism on $I$ then $d=0$.

Proof. By above theorem,

$$
\begin{aligned}
d(x)=0 \quad \forall x \in I & \\
\text { replacexbynx } & \\
d(n x) & =0 \\
\alpha(n) d(x)+d(n) x & =0 \\
d(n) x & =0 \\
\text { Replacexbymx } & \\
d(n) m x & =0 \quad \forall m \in S \quad \text { and } x \in I \\
d(n) S x & =0 \\
d(n) S I & =0 \quad \forall n \in S \\
\text { ByprimenessI } & =0 \quad \text { or } \quad d(n)=0 \\
\text { SinceIisnonzero, } d & =0
\end{aligned}
$$

\section{References}

[1] M. Chandramouleeswaran, V. Thiruveni, On derivations of semirings, Advances in Algebras, 3 (2010), 123-131. 
[2] M. Chandramouleeswarn, V. Triveni, A note on $\alpha$ derivations in semirings, International Journal of Pure and Applied Science and Technology (2011), 71-77.

[3] Jonathan S. Golan, Semirings and their Applications, Kluwer Academic Press (1969).

[4] Mustafa Kazaz, Akin Alkan, Two-sided $\Gamma-\alpha$-derivations in Prime and semiprime $\Gamma$-near-rings, Commun. Korean Math. Soc., 23, No. 4 (2008), 469-477.

[5] E.C. Posner, Derivations in prime rings, Proc. Amer. Math. Soc., 8 (1957), 1093-1100.

[6] H.S. Vandiver, Note on a simple type of algebra in which the cancellation law of addition does not hold, Bull. Amer. Math. Soc., 40 (1934), 916-920. 